\title{
Formulation of the Settling Velocity of Small Particles Initially Situated inside an Inclined Vortex
}

\author{
Urbano Sánchez \\ Fluid Mechanics Division, University of Huelva, Campus La Rábida, Carretera Huelva-Palos s/n, \\ Palos de la Frontera, 21819 Huelva, Spain \\ Correspondence should be addressed to Urbano Sánchez; urbano.sanchez@dcaf.uhu.es
}

Received 16 December 2015; Revised 15 February 2016; Accepted 28 February 2016

Academic Editor: Jerzy Bałdyga

Copyright (C) 2016 Urbano Sánchez. This is an open access article distributed under the Creative Commons Attribution License, which permits unrestricted use, distribution, and reproduction in any medium, provided the original work is properly cited.

Both the estimation of the time that small heavy particles remain inside a $3 \mathrm{D}$ vortex and the estimation of the average settling velocity of those particles are some important features in many practical situations. Previous works focused on the case of a horizontal 2D vortex. In this paper, we simulate the dynamics of heavy particles initially situated inside a three-dimensional vortex obtaining a formula for their average settling velocity. In a previous paper we obtained the trajectories of the particles and a formula that provides the time that they need to escape, $T_{e}^{*}$. This work simulates and analyses the escape process, and its main result is the obtaining, from numerical simulation, of a theoretical formulation of the average settling velocity $\left\langle V_{z}^{*}\right\rangle$ and its relationship with the elapsed time. We prove that the permanence time is of the order of $d_{p}^{*-10}$ (with $d_{p}^{*}$ particle diameter) and that the average settling velocity is of the order of $\left\langle T_{e}^{*}\right\rangle^{-1 / 5}$ for sufficiently small particles. Some applications of the settling velocity formula developed in this work would be the design of mixture devices, the design of particle separation devices, and the prediction of the settling of pollutant particles, seeds, and pollen.

\section{Introduction}

It is a proved fact that the most important flow structures in connection with suspended particles are vortices [1-3] because they can trap particles temporarily, carry them along over considerable distances, and fling them out. It is well known that heavy particles with small inertia may remain inside a vortex for very long time. Situations like that appear, for example, in turbulent flows where elongate tubes of intense vorticity exist, which have an average life time of the order of the integral time scale [4].

The knowledge of the permanence time of heavy particles inside a $3 \mathrm{D}$ vortex is very important in many areas of science and technology. For example, it can be an important feature to determine the validity of a PIV system to visualize vortical flows. Moreover, the presence of suspended particles is habitual in many natural turbulent flows. In the atmosphere, the permanence times of various aerosols determine how long those aerosols remain suspended in air.

The average settling velocity is a suitable condition to design mixture devices or particle separation devices and can provide important information about the settling of seeds, pollen particles, and pollutant particles.

The permanence time and the average settling velocity are also important because they govern the residence times of dust particles from wind erosion or manmade pollutants [5, $6]$ and the settling in estuaries of rivers [7, 8]. Moreover, they are transcendent for those cases, as small water droplets in clouds, combustion, ash from volcanic eruptions, and many industrial and environmental processes.

The trapping and settling of particles by vortical flows have been studied in the last decades. Stommel [9] showed that particles without inertia in $2 \mathrm{D}$ periodic flows may remain suspended and describe closed orbits. Auton [10] demonstrated that bubbles released beneath an irrotational vortex may become trapped at the centre of the vortex and this mechanism has been used by Sene et al. [11] to investigate the transport of bubbles in free-shear layers. Maxey and Corrsin [12] proved that aerosol particles in randomly oriented cellular flows can be trapped only if the effect of the particle inertia is neglected. However, the work of McLaughlin [13] demonstrates the possibility of suspension 
of heavy inertial particles in two-dimensional periodic flows, showing also chaotic behaviour in certain parametrical range. Ruetsch and Meiburg [14] carried out a numerical simulation of the motion of small bubbles in two-dimensional vertical flows. They found that the rate of entrapment of the bubbles into the vortex has an optimum value for some value of the Stokes number. Marcu et al. [15] made a linear stability analysis for the motion of heavy particles in a Burgers vortex. They found that in the absence of gravity sufficiently light particles can be stably located at the vortex centre, since the inward drag force created by the strain field is strong enough to overcome the destabilizing centrifugal force on the particle. A particle with a Stokes number larger than the critical value orbits the centre of the vortex along a circular trajectory. When the effect of gravity is not considered also, the vortex centre no longer is an equilibrium point, and instead either one or three equilibrium points appear away from the centre. Dávila and Hunt [3] established the dimensionless parameters that govern the movement of the particles and they studied the dynamics of heavy particles near a horizontal vortex and in turbulence under gravity. They enlarged upon some works of Crowe et al. [16] and Clifford et al. [17]. Hill [18] calculated droplet velocities, concentrations, and geometric collision rates for droplets falling into a Burgers vortex. He suggests that acceleration induced coalescence is most significant for droplets that are entrained into or formed within an intensifying vortex for droplets that are falling toward the vortex.

A common characteristic of some of the studies abovementioned is the fact of the particle ejection from the vortex centre [19] and the formation of zones of high particle concentration, as showed by numerical simulations of GañánCalvo and Lasheras [20] and Martin and Meiburg [21].

One of the most important papers relative to the trapping of particles in a line vortex is that of Fung [22]. In it, Fung studied the residence time of inertial particles in the restricted case of a two-dimensional vortex. He found that the residence time is a function of the particle inertia, its fall velocity, and the vortex's Reynolds number.

Finally, in a previous paper [23] we generalized the results mentioned above to the case of a three-dimensional vortex, obtaining a formula for the escape time of heavy particles from a $3 \mathrm{D}$ vortex.

Now, in this work we develop a formulation of the average settling velocity, the relationship between the average settling velocity and the permanence time, and the dependence on the particle diameter.

\section{Material and Methods}

To evaluate the average settling velocity of particles initially situated inside a vortex we have used the procedure known as CFD (Computational Fluid Dynamics); we have developed a computer algorithm that simulates the motion of the particles in the presence of a vortex and that calculates that velocity. After that calculation, we have carried out an asymptotic analysis to relate the average settling velocity with the average escape time.
2.1. Velocity Field. In the same way as in our previous paper [23], although there are some models of vortices, LambOseen, Burgers-Rott, Kaufmann, Sullivan, and so on, which enable the investigation of the interaction between particles and vortices through numerical procedures, we have looked for one to simulate adequately the flow field of a vortex with a relatively simple formulation according to our purpose. In this sense, we have avoided those models with components longitudinal and radial of the velocity as they would represent only an unnecessary complication and a significant increasing of the computation time but they are not contributing anything relevant to the subject of this investigation. For those reasons and for the fact that some works related with this research have used the Rankine vortex, this has been the model used in this study. The normalized 3D velocity field in Cartesian coordinates relative to the axis of the vortex is given by

$$
\begin{aligned}
& u_{x}^{*}=\frac{-2 y^{*} \Gamma^{*}}{\left(R_{v}^{* 2}+x^{* 2}+y^{* 2}\right)}, \\
& u_{y}^{*}=\frac{2 x^{*} \Gamma^{*}}{\left(R_{v}^{* 2}+x^{* 2}+y^{* 2}\right)}, \\
& u_{z}^{*}=0
\end{aligned}
$$

where $\Gamma^{*}$ is the circulation of the vortex and $R_{v}^{*}$ the radius of the vortex so the maximum value of the velocity $U^{*}$ is given on $r^{*}=R_{v}^{*}=\Gamma^{*} / U^{*}$.

The inclination of the vortex is defined by the azimuthal angle $\theta$, that is, the angle between the axis of the vortex and the fixed $Z$ axis, which is antiparallel to the direction of gravity.

2.2. Equation for Particle Motion. Although the concepts of this section are not new, we think it is suitable to include them to explain with clarity the reasons to use these equations.

Assuming that the particles have a spherical shape, that the particle-particle interactions are negligible, that is, dilute regime assumption, and that the presence of particles in the fluid does not affect the structure of the flow (this assumption is termed the one-way coupling assumption, referring to the fact that the fluid affects the particle momentum, but the particles do not influence the momentum of the fluid; this assumption is reasonable in the case of dilute regime assumption), the only forces considered are the forces from the fluid-particle interactions and the gravity effect (or buoyancy force).

Therefore, the dynamics of the particle is governed by the Maxey-Riley Equation [24], the general form of which is presented in this section:

\section{PARTICLE ACCELERATION}

$$
=\text { BUOYANCY FORCE }
$$

+ PRESSURE GRADIENT FORCE

+ VIRTUAL MASS + STOKES DRAG

+ BASSET HISTORY TERM. 
The equation, in terms of mass, reads

$$
\begin{aligned}
m_{p}^{*} \frac{d \vec{v}^{*}}{d t^{*}}= & \left(m_{p}^{*}-m_{F}^{*}\right) \vec{g}^{*}+m_{F}^{*} \frac{D \vec{u}^{*}}{D t^{*}} \\
& -\frac{1}{2} m_{F}^{*} \frac{d}{d t^{*}}\left[\vec{v}^{*}-\vec{u}^{*}-\frac{1}{10} R_{p}^{* 2} \nabla^{2} \vec{u}^{*}\right] \\
& -6 \pi R_{p}^{*} \mu^{*} \vec{Y}^{*} \\
& -6 \pi R_{p}^{* 2} \mu^{*} \int_{0}^{t^{*}} d \tau^{*} \frac{d \vec{Y}^{*} / d \tau^{*}}{\sqrt{\pi v^{*}\left(t^{*}-\tau^{*}\right)}}
\end{aligned}
$$

with $\vec{Y}^{*}=\vec{v}^{*}-\vec{u}^{*}-(1 / 6) R_{p}^{* 2} \nabla^{2} \vec{u}^{*}$ and $\vec{v}^{*}$ particle velocity, $\vec{u}^{*}$ local fluid velocity, $t^{*}$ time, $m_{p}^{*}$ particle mass, $m_{F}^{*}$ mass of the fluid displaced by the particle, $\vec{g}^{*}$ gravitational acceleration, $R_{p}^{*}$ particle radius, $\mu^{*}$ fluid viscosity, and $v^{*}$ fluid kinematic viscosity (the stars denote dimensional variables).

Assuming the particle diameter is small compared to the flow scale, the Faxen correction terms can be neglected (indeed, a small particle diameter implies that the velocity curvature has negligible effect on the calculation of the drag force on the sphere at low particle Reynolds number, which is what Faxen correction terms stand for).

Equation (3) is valid for low particle Reynolds number and it is rather complicate. In order to simplify it, the Basset History Term will be neglected (Hill [18] keeps this term for cloud droplets falling into a Burgers vortex) for various reasons: this term is expected to increase the drag force when the particle is accelerating or decelerating or when the Reynolds number for the relative motion is low [25]. However, some works [26-28] have demonstrated that the history effects are less important as originally thought because the Basset History Term initially decays at $t^{-1 / 2}$ and later at $t^{-2}$ and departure from Stokes flow lessens the history effect. Moreover, the Basset History Term is proportional to the density ratio $\rho_{p}^{*} / \rho_{F}^{*}$, so the heavier the particle, the smaller this term. A full justification of the neglecting of the mentioned term can be found in the works of Goater [29]. Given that this paper focuses on the case of heavy particles, the Basset History Term will be neglected so (3) reads

$$
\begin{aligned}
\left(m_{p}^{*}+\frac{1}{2} m_{F}^{*}\right) \frac{d \vec{v}^{*}}{d t^{*}}= & \left(m_{p}^{*}-m_{F}^{*}\right) \vec{g}^{*} \\
& +6 \pi R_{p}^{*} \mu^{*}\left[\vec{u}^{*}-\vec{v}^{*}\right]+\frac{3}{2} m_{F}^{*} \frac{\partial \vec{u}^{*}}{\partial t^{*}} \\
& +m_{F}^{*} \vec{u}^{*} \cdot \nabla \vec{u}^{*}+\frac{1}{2} m_{F}^{*} \vec{v}^{*} \cdot \nabla \vec{u}^{*}
\end{aligned}
$$

Finally, for heavy particles with $\rho_{p}^{*} \gg \rho_{F}^{*}$, that is, $m_{p}^{*} \gg m_{F}^{*}$ (with $\rho_{p}^{*}$ particle density and $\rho_{F}^{*}$ fluid density), the equation of motion is

$$
m_{p}^{*} \frac{d \vec{v}^{*}}{d t^{*}}=6 \pi R_{p}^{*} \mu^{*}\left(\vec{u}^{*}-\vec{v}^{*}\right)+m_{p}^{*} \vec{g}^{*} .
$$

The determination of the particle motion from this equation is a complex problem due to the nonlinearity introduced by the velocity field and we have to obtain computational solutions. To integrate (5) it is necessary to write it in dimensionless form introducing the suitable dimensionless parameters. If particles are sufficiently small (i.e., if the Reynolds number of the particles, based on their diameter and their terminal velocity, is small), only two dimensionless parameters are enough to describe the motion of small spherical particles: a gravitational and an inertial parameter. The gravitational parameter is the ratio of the terminal velocity of the particles in still flow $V_{t}^{*}$ to the characteristic velocity of the fluid $U^{*}=\Gamma^{*} / R_{v}^{*}: V_{t}=V_{t}^{*} / U^{*}=$ $m_{p}^{*} g^{*} / 6 \pi R_{p}^{*} \mu^{*}$. For the inertial parameter, Dávila and Hunt [3] showed that the average properties of small particles trapped in regions of concentrated vorticity (in which case the characteristic velocity of the particles is that of the fluid) can be characterised by the Stokes number defined as $S_{t}=\tau_{p}^{*} / \tau_{r}^{*}$, where $\tau_{p}^{*}=m_{p}^{*} / 6 \pi R_{p}^{*} \mu^{*}=V_{t}^{*} / g^{*}=\beta d_{p}^{* 2} /\left(18 v^{*}\right)$ is the viscous response time of the particles and $\tau_{r}^{*}=R_{v}^{*} / U^{*}=$ $R_{v}^{* 2} / \Gamma^{*}$ is the characteristic residence time of the fluid flow. Here $\beta$ is the ratio between the particle density and the fluid density and $d_{p}^{*}$ is the particle diameter. This is the case of turbulent flows, if the time that the particles need to escape from regions of intense vorticity is much greater than the life time of those vortex structures. The Stokes number determines the importance of the particle inertia such that when $S_{t}$ is very small, inertial effects are small. Considering the dimensionless variables

$$
\begin{aligned}
& \vec{x}=\frac{\vec{x}^{*}}{R_{v}^{*}}, \\
& \vec{v}=\frac{\vec{v}^{*}}{U^{*}}=\frac{\vec{v}^{*}}{\left(\Gamma^{*} / R_{v}^{*}\right)}=\frac{\vec{v}^{*} R_{v}^{*}}{\Gamma^{*}}, \\
& t=\frac{t^{*} U^{*}}{R_{v}^{*}}=\frac{t^{*}\left(\Gamma^{*} / R_{v}^{*}\right)}{R_{v}^{*}}=\frac{t^{*} \Gamma^{*}}{R_{v}^{* 2}},
\end{aligned}
$$

the scaled nondimensional equations of motion are

$$
\begin{aligned}
& \frac{d \vec{x}(t)}{d t}=\vec{v}(t) \\
& \frac{d \vec{v}(t)}{d t}=\left(\frac{1}{S_{t}}\right)\left[\vec{u}(\vec{x}, t)-\vec{v}(t)+\vec{V}_{T}\right]
\end{aligned}
$$

where the variables without stars represent dimensionless variables.

The trajectories of particles are obtained integrating (7a) and (7b) using a fourth-order variable step size Runge-Kutta method. The initial condition at the position $\vec{x}_{0}$ is that the particles have the velocity of the fluid at $\vec{x}_{0}$.

Therefore the dimensionless process is used only for calculation purposes and the results are showed in terms of dimensional variables, with tangibles magnitudes due to their practical utility.

2.3. Equilibrium Points and Trapping Region of the Vortex. In order to calculate the average settling velocity of particles initially situated inside the vortex, it is necessary to determine 
the trapping region of the vortex to take a mesh of particle in that region. For that purpose, we have to notice that the trajectories of the particles are characterised by the position of the equilibrium points of the projection of the movement over the plane perpendicular to the axis of the vortex (defined by (5)), where $d \vec{x}^{*} / d t^{*}=d \vec{v}^{*} / d t^{*}=0$. The position of those equilibrium points in the case of an irrotational vortex was obtained by Raju and Meiburg [30]; they are obtained from

$$
\vec{v}_{t}^{*}+\vec{u}^{*}\left(\vec{X}_{E}^{*}\right)=0 .
$$

Introducing in (8) the velocity field of the Rankine vortex and splitting it in two scalar equations,

$$
\begin{array}{r}
\frac{-\Omega^{*} y_{E}^{*}}{1+x_{E}^{* 2} / R_{v}^{* 2}+y_{E}^{* 2} / R_{v}^{* 2}}=0 \Longrightarrow \\
y_{E}^{*}=0, \\
-V_{t}^{*} \sin \theta+\frac{\Omega^{*} x_{E}^{*}}{1+x_{E}^{* 2} / R_{v}^{* 2}+y_{E}^{* 2} / R_{v}^{* 2}}=0
\end{array}
$$

with $\Omega^{*}=2 \Gamma^{*} / R_{v}^{* 2}$ twice the angular velocity of the vortex. Substituting (10) in (9) and performing algebraic operations this yields the following equation:

$$
\left(V_{t}^{*} \sin \theta\right) x_{E}^{* 2}-R_{v}^{* 2} \Omega x_{E}^{*}+R_{v}^{* 2} V_{t}^{*} \sin \theta=0 .
$$

The solution of (11) is

$$
x_{E}^{*}=\frac{R_{v}^{* 2} \Omega^{*} \pm \sqrt{R_{v}^{* 4} \Omega^{* 2}-4 R_{v}^{* 2} V_{t}^{* 2} \sin ^{2} \theta}}{2 V_{t}^{*} \sin \theta} .
$$

Equation (11) has no solution if $R_{v}^{* 4} \Omega^{* 2}-4 R_{v}^{* 2} V_{t}^{* 2} \sin ^{2} \theta<0$ and it has two real solutions if $R_{v}^{* 4} \Omega^{* 2}-4 R_{v}^{* 2} V_{t}^{* 2} \sin ^{2} \theta>0$ :

$$
\begin{aligned}
x_{E 1}^{*} & =\frac{\Omega^{*}-\sqrt{\Omega^{* 2}-4 V_{t}^{* 2} \sin ^{2} \theta / R_{v}^{* 2}}}{2 V_{t}^{*} \sin \theta / R_{v}^{* 2}} \\
& =\frac{\Gamma^{*}-\sqrt{\Gamma^{* 2}-R_{v}^{* 2} V_{t}^{* 2} \sin ^{2} \theta}}{V_{t}^{*} \sin \theta}, \\
x_{E 2}^{*} & =\frac{\Omega^{*}+\sqrt{\Omega^{* 2}-4 V_{t}^{* 2} \sin ^{2} \theta / R_{v}^{* 2}}}{2 V_{t}^{*} \sin \theta / R_{v}^{* 2}} \\
& =\frac{\Gamma^{*}+\sqrt{\Gamma^{* 2}-R_{v}^{* 2} V_{t}^{* 2} \sin ^{2} \theta}}{V_{t}^{*} \sin \theta} .
\end{aligned}
$$

$\vec{X}_{E 1}^{*}=\left(X_{E 1}^{*}, 0\right)$ is an unstable focus of the dynamic system defined by the equations of motion and $\vec{X}_{E 2}^{*}=\left(X_{E 2}^{*}, 0\right)$ is a saddle point. When $V_{t}^{*} \rightarrow 0, \vec{x}_{E 1}^{*} \rightarrow(0,0)$, and $\vec{x}_{E 2}^{*} \rightarrow\left(R_{v}^{* 2} \Omega^{*} / V_{t}^{*} \sin \theta, 0\right)$, the limit trajectories starting at the saddle point enclose a region that cannot be reached by particles coming from above the vortex: trapping region. is, if

Equation (11) has only one solution if the filing is zero, that

$$
R_{v}^{* 4} \Omega^{* 2}-4 R_{v}^{* 2} V_{t}^{* 2} \sin ^{2} \theta=\Gamma^{* 2}-R_{v}^{* 2} V_{t}^{* 2} \sin ^{2} \theta=0 ;
$$

that is the case in which the equilibrium points condense in one point. From (14) it is possible to deduce the minimum ratio of the trajectories of the particles around the vortex $\left(R_{v}^{*}\right)_{\min }$ which is the characteristic length scale of this problem:

$$
\begin{aligned}
\left(R_{v}^{*}\right)_{\min }^{2} V_{t}^{* 2} \sin ^{2} \theta & =\Gamma^{* 2} \Longrightarrow \\
\left(R_{v}^{*}\right)_{\min } & =\frac{\Gamma^{*}}{V_{t}^{* 1}}=L_{c}^{*} .
\end{aligned}
$$

As we have already commented, there are some trajectories that begin at the saddle point and enclose a region that cannot be achieved by particles coming from above the vortex, the limit trajectories. These trajectories are useful, as it will be seen later, for determining the trapping region of the vortex and for taking a mesh of particles in that region to calculate the average settling velocity of particles.

The distance between the equilibrium points is given by

$$
\frac{\left|\vec{x}_{E 2}^{*}-\vec{x}_{E 1}^{*}\right|}{2}=\frac{\sqrt{\Gamma^{* 2}-R_{v}^{* 2} V_{t}^{* / 2}}}{V_{t}^{* \prime}} .
$$

From (15) and (16) it is clear that when $V_{t}^{* \prime} \rightarrow 0$, $\left|\vec{x}_{E 2}^{*}-\vec{x}_{E 1}^{*}\right| / 2 \rightarrow L_{c}^{*}$.

It is advantageous to carry out the development to obtain the equilibrium points and the characteristic length scale in terms of nondimensional variables. The results are

$$
\begin{aligned}
x_{E 1} & =\frac{1-\sqrt{1-V_{t}^{2} \sin ^{2} \theta}}{V_{t} \sin \theta}=\frac{1-\sqrt{1-V_{t}^{\prime 2}}}{V_{t}^{\prime}}, \\
x_{E 2} & =\frac{1+\sqrt{1-V_{t}^{2} \sin ^{2} \theta}}{V_{t} \sin \theta}=\frac{1+\sqrt{1-V_{t}^{\prime 2}}}{V_{t}^{\prime}}, \\
\left|\vec{x}_{E 2}-\vec{x}_{E 1}\right| & =\frac{2 \sqrt{1-V_{t}^{\prime 2}}}{V_{t}^{\prime}}, \\
L_{c} & =\frac{1}{V_{t}^{\prime}} .
\end{aligned}
$$

From (19) and (20) $\left|\vec{x}_{E 2}-\vec{x}_{E 1}\right| / 2 \rightarrow L_{c}$ when $V_{t}^{\prime} \rightarrow 0$.

\section{Results and Discussion}

The main objective of this work is to study the relationship between the average permanence time $\left\langle T_{e}^{*}\right\rangle$ and the average settling velocity $\left\langle V_{z}^{*}\right\rangle$. In this way, we have taken a mesh of 500 uniformly distributed particles situated in the trapping region of the vortex (limited by the limit trajectories; see Figure 1) and we have calculated the average values of the permanence time and the settling velocity, defined as

$$
\begin{aligned}
& \left\langle T_{e}^{*}\right\rangle=\frac{1}{N} \sum_{i=1}^{i=N} T_{e_{i}}^{*}, \\
& \left\langle V_{z}\right\rangle=\frac{1}{N} \sum_{i=1}^{i=N} \frac{Z_{i t}-Z_{i 0}}{t}
\end{aligned}
$$




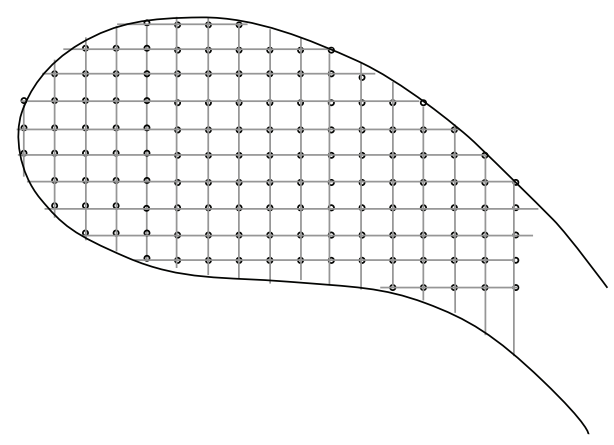

FIGURE 1: Mesh of particles within the trapping region to obtain average values of the permanence time and the settling velocity.

with $T_{e_{i}}^{*}$ being the permanence time of a particle, $Z_{i 0}$ being the initial $Z$ coordinate of a particle, $Z_{i t}$ being the final $Z$ coordinate of the particle at the time $t$, and $N$ being the number of particles. The time $t$ is large enough to reach the asymptotic values of $\left\langle V_{z}\right\rangle$. Averages are not made over the orientation angle; they are made without loss of generality as we have already commented for $\theta=\pi / 4$. Other values for $\theta$ only provide quantitative rather than qualitative changes in results.

To determine the mesh of particles it is necessary to obtain the differential equation of the limit trajectory, integrate that differential equation to obtain the algebraic equation, and solve that algebraic equation. The limit trajectory is determined by

$$
\begin{aligned}
& \frac{d x^{*}}{d t^{*}}=u_{x}^{*}, \\
& \frac{d y^{*}}{d t^{*}}=u_{y}^{*}-V_{t}^{\prime *} .
\end{aligned}
$$

The integration of these equations (introducing the Rankine velocity field) yields

$$
\begin{aligned}
& y^{*} \\
& =\sqrt{\left(x_{0}^{* 2}+y_{0}^{* 2}+R_{v}^{* 2}\right) e^{\left(2 V_{t}^{\prime *} / \Omega^{*} R_{v}^{* 2}\right)\left(x^{*}-x_{0}^{*}\right)}-x^{* 2}-R_{v}^{* 2}} \\
& =\sqrt{\left(x_{0}^{* 2}+y_{0}^{* 2}+R_{v}^{* 2}\right) e^{\left(V_{t}^{\prime *} / \Gamma^{*}\right)\left(x^{*}-x_{0}^{*}\right)}-x^{* 2}-R_{v}^{* 2}},
\end{aligned}
$$

where $x_{0}^{*}, y_{0}^{*}$ are the coordinates of a known point of the limit trajectory, that is, the saddle point. Equation (23) can be written in nondimensional form as

$$
\begin{aligned}
y & =\sqrt{\left(x_{0}^{2}+y_{0}^{2}+1\right) e^{V_{t}^{\prime}\left(x-x_{0}\right)}-x^{2}-1} \\
& =\sqrt{\left(x_{0}^{2}+y_{0}^{2}+1\right) e^{V_{t} \sin \theta\left(x-x_{0}\right)}-x^{2}-1} .
\end{aligned}
$$

It is not possible to obtain the analytical solution of (23) or (24). It is necessary to solve them through an iterative process.

The solutions are shown in Table 1 and Figure 2 and in Table 2 and Figure 3, respectively.

In Table 2, as in Table 1, the limit values of the mesh of particles $x_{i}, x_{f}$ in the trapping region of the vortex are
TABLE 1: Limit dimensionless values for the mesh of particles in the trapping region of a Rankine vortex as a function of $V_{t}$.

\begin{tabular}{lcc}
\hline$V_{t}$ & \multicolumn{2}{c}{ Limit values } \\
\hline 0,24 & $x_{i}$ & $x_{f}$ \\
0,22 & $-2,028$ & 5,468 \\
0,20 & $-2,243$ & 5,994 \\
0,18 & $-2,497$ & 6,623 \\
0,16 & $-2,805$ & 7,388 \\
0,15 & $-3,187$ & 8,340 \\
0,14 & $-3,414$ & 8,910 \\
0,13 & $-3,672$ & 9,561 \\
0,12 & $-3,970$ & 10,310 \\
0,11 & $-4,315$ & 11,183 \\
0,10 & $-4,723$ & 12,214 \\
0,09 & $-5,210$ & 13,450 \\
0,08 & $-5,804$ & 14,958 \\
0,07 & $-6,544$ & 16,842 \\
0,06 & $-7,491$ & 19,263 \\
0,05 & $-8,758$ & 22,487 \\
0,04 & $-10,526$ & 27,000 \\
0,03 & $-13,173$ & 33,763 \\
0,02 & $-17,581$ & 45,035 \\
0,008 & $-26,389$ & 65,570 \\
0,006 & $-66,003$ & 168,953 \\
& $-88,007$ & 225,275 \\
\hline
\end{tabular}

shown. These values are used in the calculations that, as we have already mentioned, are performed in dimensionless form. For that reason, these values of $x_{i}$ and $x_{f}$ are shown in dimensionless form. Clearly these limit values depend on the value of the particle diameter $d_{p}^{*}$ and the circulation of the vortex $\Gamma^{*}$ and this dependence is shown in Table 2.

The results for $\left\langle T_{e}^{*}\right\rangle$ and $\left\langle V_{z}^{*}\right\rangle$ can be seen in Figures 4 and 5. In these figures we present the results in dimensional form because we think it is recommendable to show them in terms of physical variables, with tangible magnitudes due to their practical utility.

Figure 4 shows the average permanence time versus the particle diameter for various values of the circulation of the vortex. The symbols represent the average permanence time obtained by means of numerical simulation and the solid lines represent the theoretical results for the average permanence time obtained in our previous work [23]. In that work we obtained through an asymptotic analysis that the average escape time of small inertial particles from the capture region of the vortex is

$$
T_{e}^{*}=K_{e} \frac{\Gamma^{* 2}}{\tau_{p}^{*} V_{t}^{* / 4}},
$$

where $K_{e}$ is a dimensionless constant whose value, from numerical calculations, is 0.036 . 
TABLE 2: Limit dimensionless values for the mesh of particles in the trapping region of a Rankine vortex as a function of $d_{p}^{*}$ and $\Gamma^{*}$.

\begin{tabular}{|c|c|c|c|c|c|c|}
\hline \multirow{3}{*}{$d_{p}^{*}(\mu \mathrm{m})$} & \multicolumn{6}{|c|}{$\Gamma^{*}\left(\mathrm{~m}^{2} / \mathrm{s}\right)$} \\
\hline & \multicolumn{2}{|c|}{$10^{-5}$} & \multicolumn{2}{|c|}{$3 \cdot 10^{-5}$} & \multicolumn{2}{|c|}{$10^{-4}$} \\
\hline & $x_{i}$ & $x_{f}$ & $x_{i}$ & $x_{f}$ & $x_{i}$ & $x_{f}$ \\
\hline 6 & $-4,77$ & 12,3 & & & & \\
\hline 7 & $-3,46$ & 9,03 & & & & \\
\hline 8 & $-2,59$ & 6,86 & $-8,14$ & 20,92 & & \\
\hline 9 & $-1,98$ & 5,35 & $-6,41$ & 16,51 & $-21,56$ & 55,22 \\
\hline 10 & $-1,52$ & 4,26 & -5.17 & 13,35 & $-17,45$ & 44,72 \\
\hline 11 & $-1,17$ & 3,44 & $-4,24$ & 11,01 & $-14,41$ & 36,94 \\
\hline 12 & $-0,88$ & 2,79 & $-3,53$ & 9,22 & $-12,10$ & 31,04 \\
\hline 13 & $-0,63$ & 2,26 & $-2,98$ & 7,82 & $-10,30$ & 26,44 \\
\hline 14 & $-0,39$ & 1,81 & $-2,53$ & 6,71 & $-8,87$ & 22,78 \\
\hline 15 & $-0,15$ & 1,40 & $-2,16$ & 5,81 & $-7,72$ & 19,83 \\
\hline 16 & 0,14 & 0,95 & $-1,86$ & 5,06 & & \\
\hline 17 & & & $-1,60$ & 4,44 & $-5,98$ & 15,42 \\
\hline 18 & & & $-1,37$ & 3,91 & & \\
\hline 19 & & & $-1,18$ & 3,46 & & \\
\hline 20 & & & $-1,00$ & 3,06 & $-4,28$ & 11,10 \\
\hline 21 & & & $-0,84$ & 2,72 & & \\
\hline 22 & & & $-0,70$ & 2,41 & & \\
\hline 23 & & & $-0,56$ & 2,13 & & \\
\hline 24 & & & $-0,43$ & 1,87 & & \\
\hline 25 & & & & & $-2,66$ & 7,03 \\
\hline 26 & & & $-0,15$ & 1,39 & & \\
\hline 30 & & & & & $-1,74$ & 4,78 \\
\hline 40 & & & & & $-0,71$ & 2,43 \\
\hline 50 & & & & & 0,07 & 1,05 \\
\hline
\end{tabular}

Recording that $\tau_{p}^{*}=\beta d_{p}^{* 2} /\left(18 v^{*}\right)$ and that $V_{t}^{*}=\tau_{p}^{*} g^{*}=$ $\beta g^{*} d_{p}^{* 2} /\left(18 v^{*}\right)$ the average permanence time can be written as a function of the particle diameter:

$$
\left\langle T_{e}^{*}\right\rangle=K_{e} \frac{18^{5} v^{* 5} \Gamma^{* 2}}{\beta^{5} g^{* 4} \sin ^{4} \theta} d_{p}^{*-10} .
$$

From Figure 4, our numerical simulation of the average permanence time follows (25) or (26) for sufficiently small particles; even a very good agreement can be observed. From the observation of Figure 4 it is clear that as the particle diameter increases the average permanence time decreases, as we can expect.

Finally, the relationship between the average permanence time and the average settling velocity can be observed with clarity in Figure 5.

Both Figures 4 and 5 have been obtained using as variables the values of the particle diameter $d_{p}^{*}$. But in both figures we have taken exactly the same values of $d_{p}^{*}$.

In Figure 5, the solid line represents again the theoretical result obtained with (25) or (26); a very good agreement between our numerical results and (25) and (26) can be

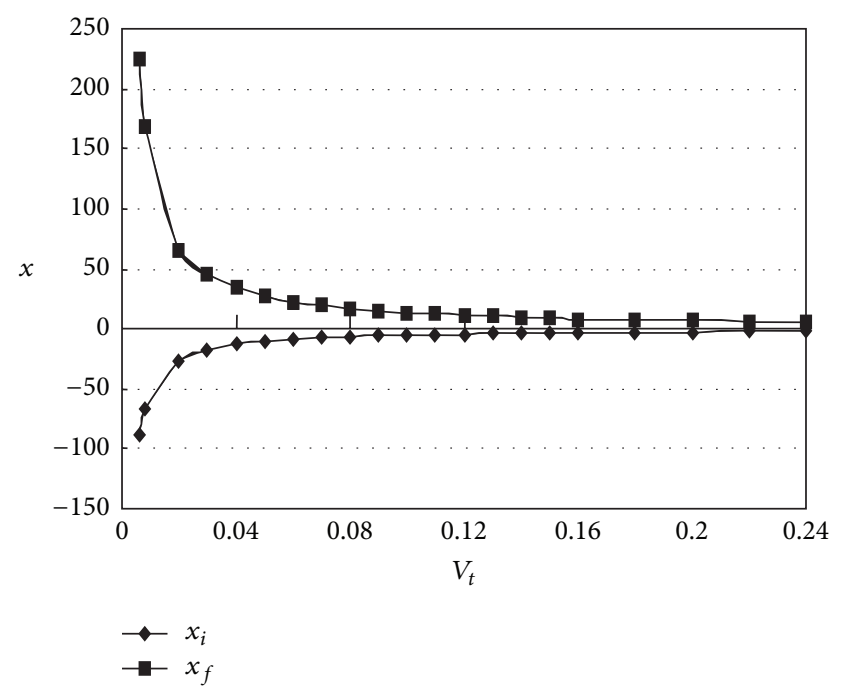

FIGURE 2: Limits of the mesh of particles inside the trapping region of the vortex as a function of $V_{t}$.

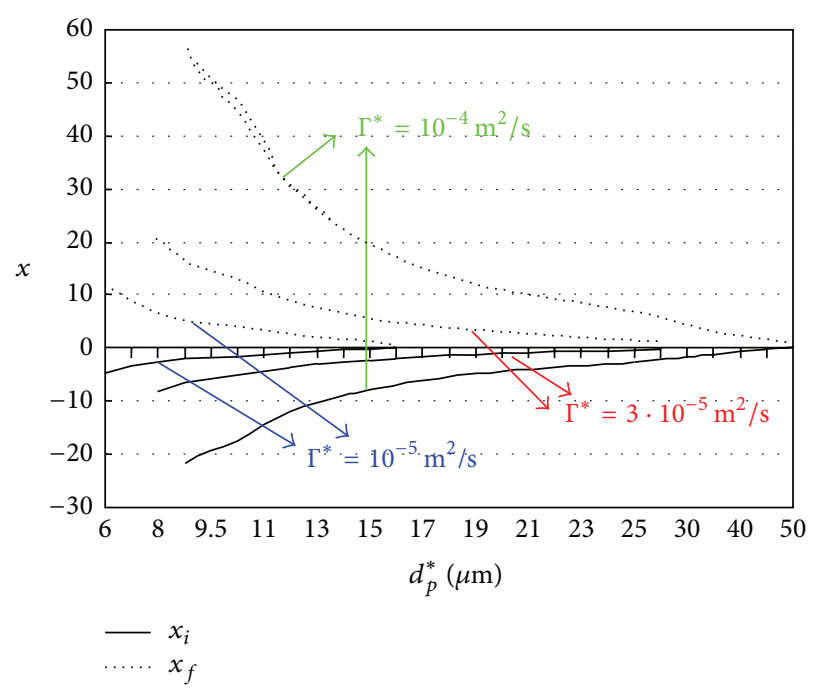

FIGURE 3: Limits of the mesh of particles inside the trapping region of the vortex as a function of $d_{p}^{*}$ and $\Gamma^{*}$.

again observed. It is clear too that when $\left\langle T_{e}^{*}\right\rangle$ increases $\left\langle V_{z}\right\rangle$ decreases.

3.1. Formulation of the Settling Velocity. An important feature can be seen from Figure 5. For large average permanence time, that is, for sufficiently small particles, the curve $\left\langle V_{z}\right\rangle-$ $\left\langle T_{e}^{*}\right\rangle$ is a straight line (in logarithmic scale). This fact suggests the possibility of developing a scaling similar to (25) or (26) for the average settling velocity of inertial particles in vortical flows. It is possible to establish the equation of the straight lines of Figure 5. In all the cases we obtained

$$
\left\langle V_{z}\right\rangle=\frac{K_{s}}{\left\langle T_{e}^{*}\right\rangle^{0.19}}
$$




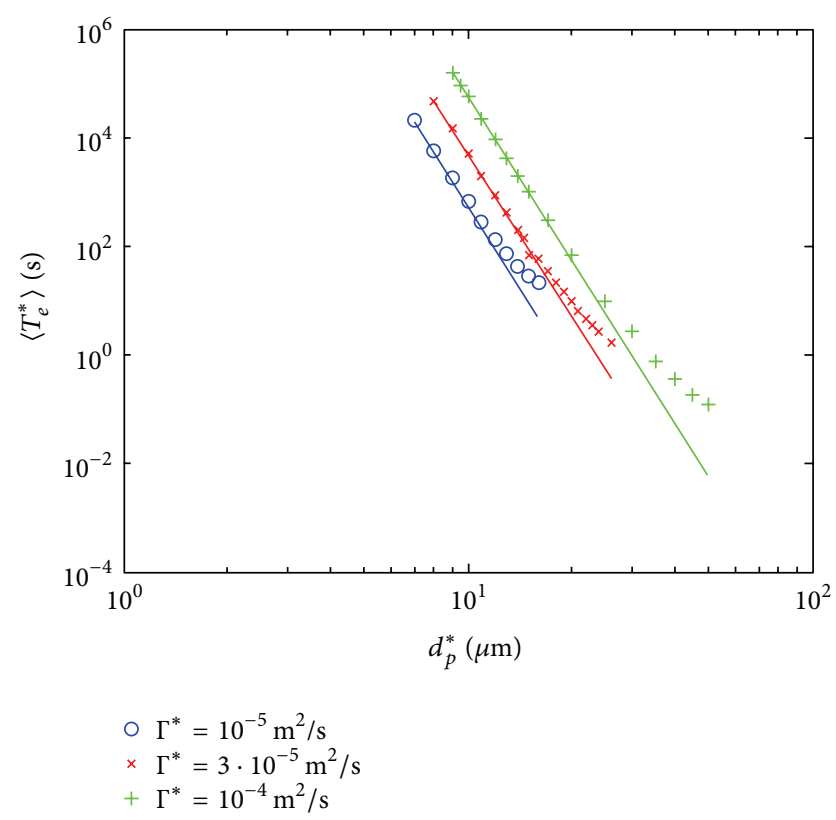

FIgURE 4: Average permanence time of particles inside an inclined vortex versus the particle diameter for various values of the circulation of the vortex and $R_{v}^{*}=0.001 \mathrm{~m}$.

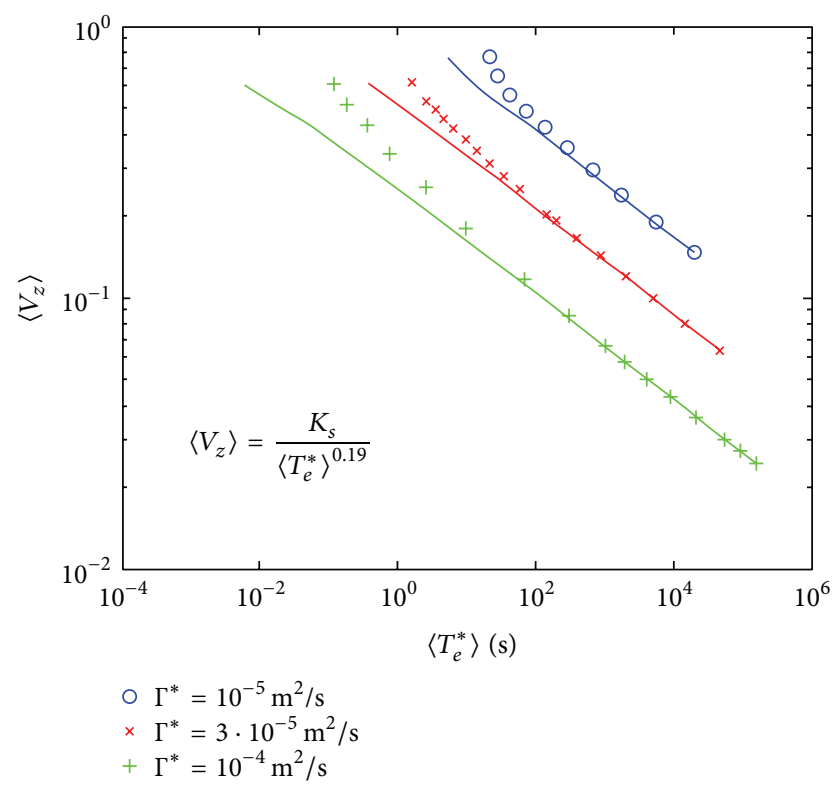

FIGURE 5: Average settling velocity of particles initially situated inside an inclined vortex versus the average permanence time for $R_{v}^{*}=0.001 \mathrm{~m}$, various values of the circulation of the vortex, and the values for the diameter of the particles as in Figure 4.

or, in dimensional terms,

$$
\begin{aligned}
\left\langle V_{z}^{*}\right\rangle & =\frac{K_{s} \Gamma^{*}}{R_{v}^{*}\left\langle T_{e}^{*}\right\rangle^{0.19}} \\
& =\frac{K_{s} \Gamma^{*}}{R_{v}^{*}}\left[K_{e} \frac{18^{5} v^{* 5} \Gamma^{* 2}}{\beta^{5} g^{* 4} \sin ^{4} \theta} d_{p}^{*-10}\right]^{-0.19},
\end{aligned}
$$

where $K_{s_{5}}$ is a constant equal to $1,0.54$, and $0.26(\mathrm{~m} / \mathrm{s})^{0.19}$ for $\Gamma^{*}=10^{-5}, 3 \times 10^{-5}$, and $10^{-4} \mathrm{~m}^{2} / \mathrm{s}$, respectively.

\section{Conclusions}

The more important result of this work is the development of a formulation for the average settling velocity of small particles initially situated inside an inclined vortex.

We carry out an analysis of the average settling velocity $\left\langle V_{z}\right\rangle$ of particles initially situated inside the trapping region of the vortex and its relationship with the average permanence time $\left\langle T_{e}^{*}\right\rangle$. We show that as the particle diameter increases $\left\langle V_{z}\right\rangle$ increases and $\left\langle T_{e}^{*}\right\rangle$ decreases and that for sufficiently small particles $\left\langle T_{e}^{*}\right\rangle$ follows the theoretical value obtained by Sánchez [23]. Moreover, our numerical simulations prove that, for sufficiently small particles, there is a relationship between $\left\langle V_{z}\right\rangle$ and $\left\langle T_{e}^{*}\right\rangle$; we prove that $\left\langle V_{z}\right\rangle$ is of the order of $\left\langle T_{e}^{*}\right\rangle^{-1 / 5}$ and we also obtain a formulation for $\left\langle V_{z}\right\rangle$. This result is important because it provides a theoretical formulation of the average settling velocity similar to that obtained by Sánchez [23] for the escape time.

\section{Competing Interests}

The author declares that there are no competing interests regarding the publication of this paper.

\section{References}

[1] A. J. Yule, "Investigations of eddy coherence in jet flows," in The Role of Coherent Structures in Modelling Turbulence and Mixing, J. Jimenez, Ed., vol. 136 of Lecture Notes in Physics, pp. 188-207, Springer, 1981.

[2] J. C. H. Fung, "Gravitational settling of small spherical particles in unsteady cellular flow fields," Journal of Aerosol Science, vol. 28, no. 5, pp. 753-787, 1997.

[3] J. Dávila and J. C. R. Hunt, "Settling of small particles near vortices and in turbulence," Journal of Fluid Mechanics, vol. 440, pp. 117-145, 2001.

[4] A. Vincent and M. Meneguzzi, "The dynamics of vorticity tubes in homogeneous turbulence," Journal of Fluid Mechanics, vol. 258, pp. 245-254, 1994.

[5] P. Nielsen, "On the motion of suspended sand particles," Journal of Geophysical Research, vol. 89, no. 1, pp. 616-626, 1984.

[6] P. F. Tooby, G. L. Wick, and J. D. Isaacs, "The motion of small sphere in a rotating velocity field: a possible mechanism for suspending particles in turbulence," Journal of the Geophysical Research, vol. 82, no. 15, pp. 2096-2100, 1977.

[7] C. G. D. Toit and J. F. A. Sleath, "Velocity measurements close to rippled beds in oscillatory flow," Journal of Fluid Mechanics, vol. 112, p. 71, 1981.

[8] R. J. Perkins and J. F. A. Sleath, "Near bed velocities in combined steady plus oscillatory flow," in Proceedings of the 20th IAHR World Congress VII, p. 173, Moscow, Russia, September 1983.

[9] H. Stommel, "Trajectories of small bodies sinking slowly through convection cells," Journal of Marine Research, vol. 8, pp. 24-29, 1949.

[10] T. R. Auton, The dynamics of bubbles, drops and particles in motion in liquids [Ph.D. dissertation], University of Cambridge, Cambridge, UK, 1983. 
[11] K. J. Sene, J. C. R. Hunt, and N. H. Thomas, "The role of coherent structures in bubble transport by turbulent shear flows," Journal of Fluid Mechanics, vol. 259, pp. 219-240, 1994.

[12] M. R. Maxey and S. Corrsin, "Gravitational settling of aerosol particles in randomly oriented cellular flow field," Journal of the Atmospheric Sciences, vol. 43, no. 11, pp. 1112-1134, 1986.

[13] J. B. McLaughlin, "Particle size effects on Lagrangian turbulence," Physics of Fluids, vol. 31, no. 9, p. 2544, 1988.

[14] G. R. Ruetsch and E. Meiburg, "On the motion of small spherical bubbles in two-dimensional vortical flows," Physics of Fluids A, vol. 5, no. 10, pp. 2326-2341, 1992.

[15] B. Marcu, E. Meiburg, and P. K. Newton, "Dynamics of heavy particles in a Burgers vortex," Physics of Fluids, vol. 7, no. 2, pp. 400-410, 1995.

[16] C. T. Crowe, T. R. Troutt, and J. N. Chung, "Particle interactions with vortices," in Fluid Vortices, S. I. Green, Ed., vol. 30 of Fluid Mechanics and Its Applications, chapter 19, pp. 829-861, Kluwer Academic, New York, NY, USA, 1995.

[17] N. J. Clifford, J. R. French, and J. Hardisty, Turbulence: Perspectives on Flow and Sediment Transport, John Wiley \& Sons, Chichester, UK, 1983.

[18] R. J. Hill, "Geometric collision rates and trajectories of clouds droplets falling into a Burgers vortex," Physics of Fluids, vol. 17, no. 3, p. 37103, 2005.

[19] M. R. Maxey, "The motion of small spherical particles in a cellular flow field," Physics of Fluids, vol. 30, no. 7, p. 1915, 1987.

[20] A. M. Gañán-Calvo and J. C. Lasheras, "The dynamics and mixing of small spherical particles in a plane, free shear layer," Physics of Fluids A, vol. 3, no. 5, pp. 1207-1217, 1991.

[21] J. E. Martin and E. Meiburg, "The accumulation and dispersion of heavy particles in forced two-dimensional mixing layers. I. The fundamental and subharmonic cases," Physics of Fluids, vol. 6, no. 3, pp. 1116-1132, 1994.

[22] J. C. H. Fung, "Residence time of inertial particles in a vortex," Journal of Geophysical Research: Oceans, vol. 105, no. 6, pp. 14261-14272, 2000.

[23] U. Sánchez, "Development and numerical validation of a formula for the escape time of heavy particles from a vortex," Research Journal of Chemistry and Environment, vol. 17, no. 4, pp. 79-87, 2013.

[24] M. R. Maxey and J. J. Riley, "Equation of motion for a small rigid sphere in a nonuniform flow," Physics of Fluids, vol. 26, no. 4, pp. 883-889, 1983.

[25] R. Clift, J. R. Grace, and M. E. Weber, Bubbles, Drops and Particles, Academic Press, New York, NY, USA, 1978.

[26] R. Mei, R. J. Adrian, and T. J. Hanratty, "Particle dispersion in isotropic turbulence under stokes drag and Basset force with gravitational settling," Journal of Fluid Mechanics, vol. 125, pp. 481-495, 1991.

[27] P. M. Lovalenti and J. F. Brady, “The force on a bubble, drop or particle in arbitrary time-dependent motion at small Reynolds number," Physics of Fluids A, vol. 5, no. 9, pp. 2104-2116, 1993.

[28] E. J. Chang and M. R. Maxey, "Unsteady flow about a sphere at low to moderate Reynolds number. Part 1. Oscillatory motion," Journal of Fluid Mechanics, vol. 277, pp. 347-379, 1994.

[29] A. Goater, Dispersion of heavy particles in an isolated pancakelike vortex [Ph.D. thesis], University of British Columbia, 2003.

[30] N. Raju and E. Meiburg, "Dynamics of small, spherical particles in vortical and stagnation point flow fields," Physics of Fluids, vol. 9, no. 2, pp. 299-314, 1997. 


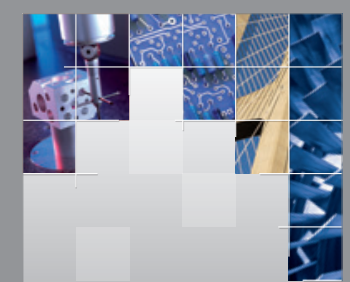

\section{Enfincering}
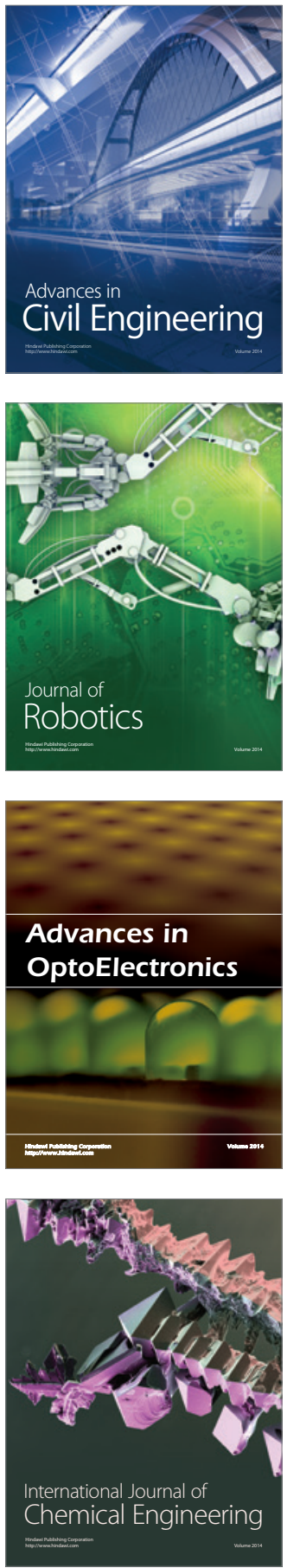

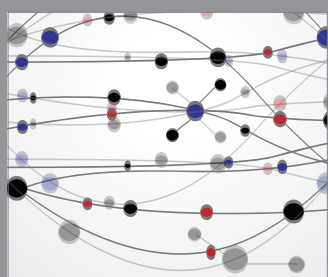

The Scientific World Journal

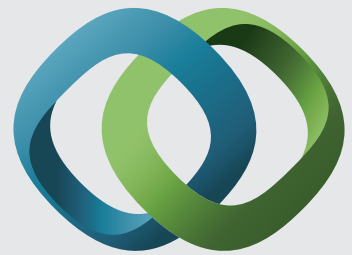

\section{Hindawi}

Submit your manuscripts at

http://www.hindawi.com
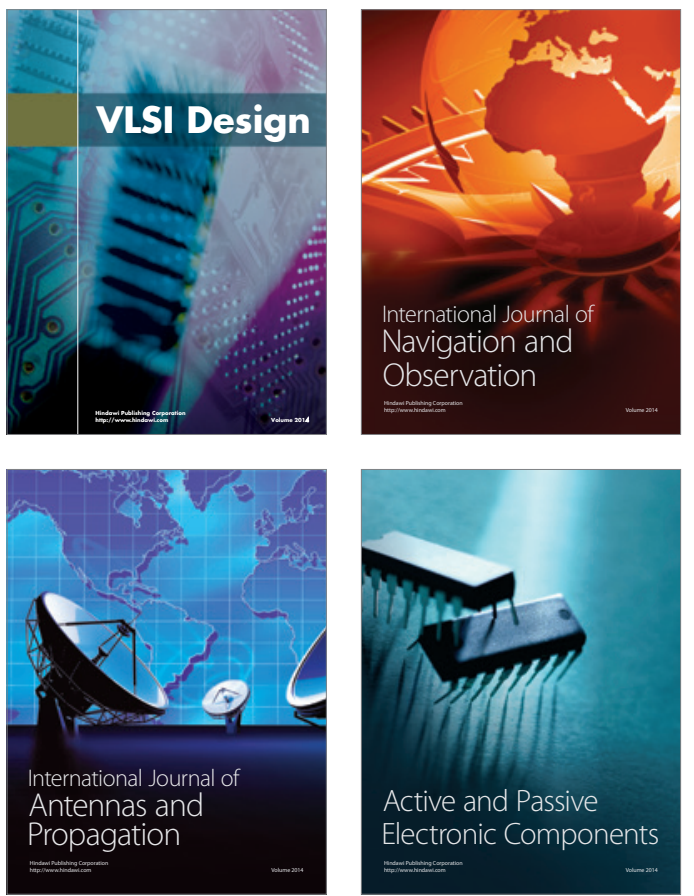
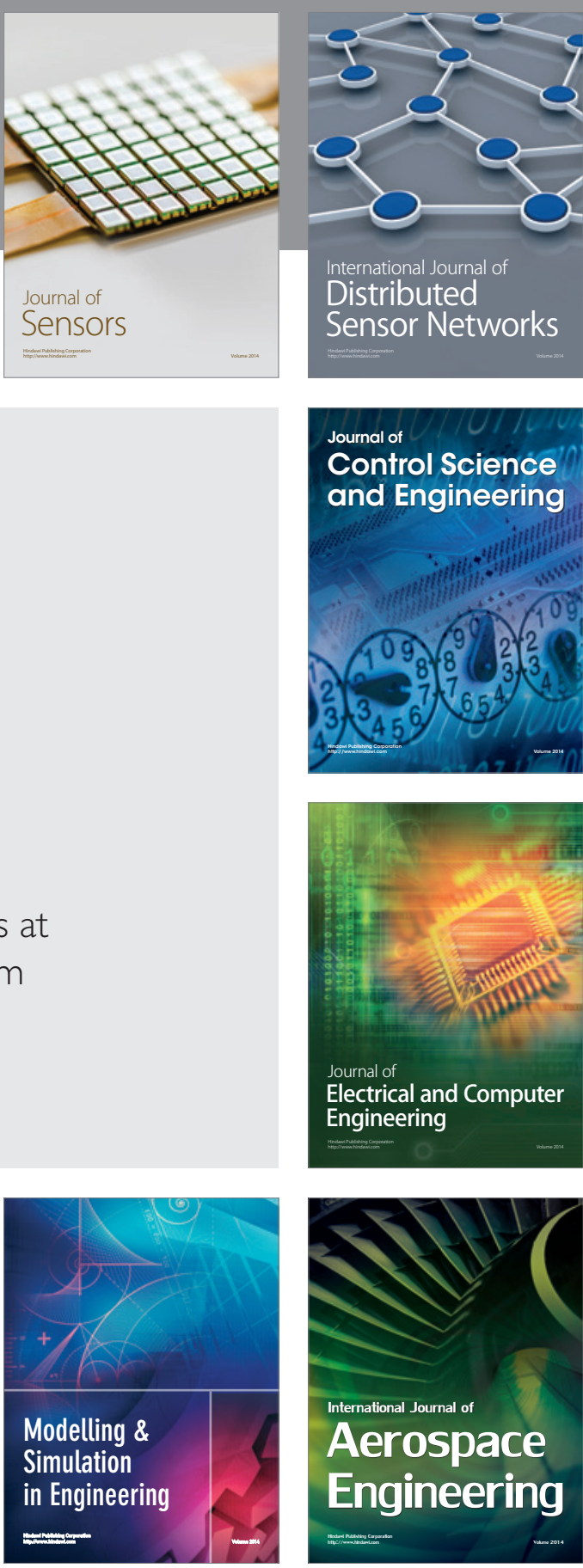

International Journal of

Distributed

Sensor Networks

Journal of

Control Science

and Engineering
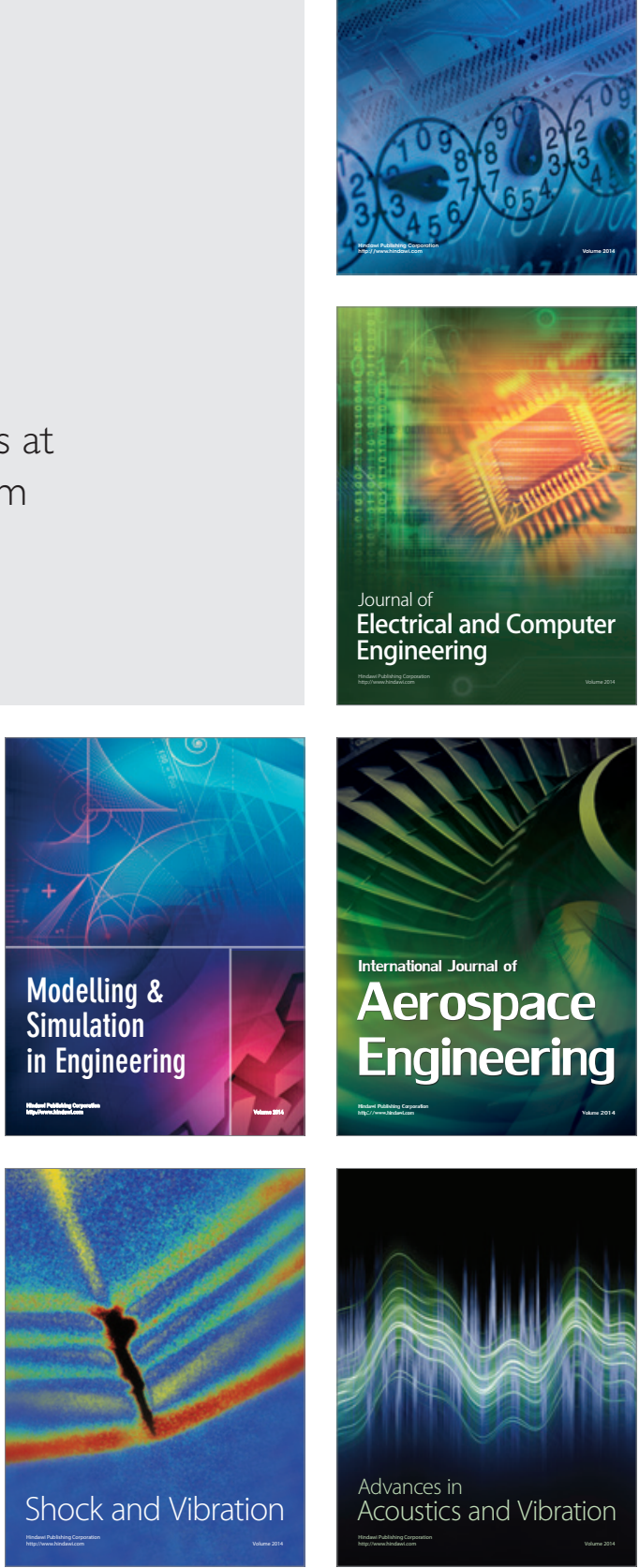